\title{
Spinning on the edge of graphene
}

The 2D form of carbon known as graphene has many potentially useful properties, but is usually not magnetic when pristine. However, theoretical predictions suggest that the edges of graphene sheets should become magnetic when they have a zigzag arrangement of carbon atoms. Observing this effect has been challenging because of the difficulties of detecting the predicted minute magnetic signal and because it is hard to fabricate defect-free edges that have the required shape. On page 691, Slota et al. report a method for making nanometre-wide graphene ribbons in solution, and thereby for producing nanoribbons with well-defined zigzag edges 'decorated' with organic radical molecules that bear electron spins - a quantum property of electrons that is associated with magnetism. The authors' results provide solid evidence of magnetism at graphene edges, and show that edge spins have potentially useful quantum dynamics. Magnetic forms of graphene would be useful for spintronics, a technology that forms the basis of today's magnetic data storage. But the main interest in generating magnetic edge states in graphene is for quantum technologies. Electron spins can adopt two orientations relative to an external magnetic field, and these could be used to encode the ' 0 ' and ' 1 ' states of a quantum bit (qubit), the basic information unit of future quantum computers and quantum-simulation devices. The quantum states of a qubit must be strongly coupled to external control stimuli that drive the qubit's operation, but they must also be isolated from random external perturbations that can irreversibly upset the 'coherent' evolution of such quantum states (coherence is the existence of non-classical correlations between quantum states). In these respects, graphene has potential advantages over other materials that are being investigated as hosts for spin qubits, such as gallium arsenide or silicon: electric currents flowing through a graphene sheet provide a means of coupling and manipulating spins; and the two main sources of decoherence are minimal in graphene. These sources of decoherence are the coupling between an electron's spin and its orbital motion (which is weak in graphene), and interactions of electron spins with atoms that have nuclear spins (the concentration of which is low in graphene). Why has it been so difficult to observe magnetic edge states experimentally? The electronic and magnetic properties of graphene nanoribbons correlate closely with the structures of their edges, and are sensitive to even minute numbers of defects. Isolating a sufficient number of nanoribbons that have perfect zigzag edges to enable their magnetic characterization is extremely challenging, and so the data from such studies6 are scarce and inconclusive. Experiments performed on single graphene layers prepared in situ under a high vacuum have revealed the formation of local electronic states at edges, but did not provide any evidence of magnetism. By expanding a previously developed chemical method, Slota et al. synthesized graphene nanoribbons in solution that have uniform widths and zigzag edges. The authors attached nitronyl nitroxide molecules - chemically robust organic radicals, which are magnetic because they carry an unpaired electron - to specific edge sites. This method produces large amounts (milligram quantities) of chemically stable graphene nanoribbons that can be studied using conventional spectroscopic techniques. The authors show that the electron spins at the radicals induce a spin density at the edge carbon sites where the radicals are bonded, and therefore induce magnetic edge states. 
This trick is akin to moving a row of corks on a string up and down on the surface of a pool to induce ordered water oscillations at the pool's edge; not only do the corks induce waves, but they also make them easier to visualize. Besides proving the existence of magnetic edge states, Slota and colleagues' experiments provide the first direct determination of the strength of the tiny spin-orbit coupling in their system. These findings will help to validate theoretical models of the electronic structure of graphene and its edge states. The authors also measured the characteristic rates at which spins relax (reach equilibrium with the graphene lattice) and the time taken for them to lose coherence. The measured decoherence times are roughly one microsecond at room temperature - which is promising, because it means that spin coherence is preserved for much longer than has previously been measured in graphene electronic devices. A plausible explanation for this is that the graphene nanoribbons are free from the structural randomness and extrinsic effects (such as spin scattering caused by connecting graphene to electrodes) that have suppressed spin coherence in other systems. Slota et al. find that decoherence in their nanoribbons seems to be mainly associated with inter actions of the electron spins with nuclear spins in the radical molecule. This is good news, because chemical methods are available to reduce the concentration of nuclear spins, or to make spin qubits insensitive to the magnetic noise generated by nuclear spins. Finally, the authors showed that unpaired electrons at the radicals interact with the edge spins. These interactions might allow graphene to be used as a coherent communication channel between different radical spins, and might therefore serve as the basis of the two-qubit logic gates necessary for a quantum computer. Slota et al. show that the attachment of magnetic molecules to graphene creates coherent magnetic states on it, nicely complementing previously reported experiments that showed how graphene influences the electron spins on molecules deposited on it. However, in the authors' system, electron spin is 'injected' into the nanoribbons from the radical molecules - so the intrinsic magnetism of graphene edges remains to be investigated. One way to explore this would be to attach non-magnetic molecules, rather than free radicals, to the graphene edges. A formidable challenge in the development of the reported nanoribbons for quantum computers will be to design a system that can manipulate and read out each qubit in a nanoribbon, and that can switch interactions between qubits on and off, in a way that also allows the computer to expand to incorporate more qubits without losing control of them. This will probably require graphene nanosheets to be coupled to a solid-state device, so it remains to be seen how the effects of coupling to the device will affect spin coherence. Moreover, if the strength of the spin-orbit coupling of edge-modified graphene nanoribbons can be increased, then the spin at the attached molecules could be manipulated using an electric field. Such strengthening might be achieved by replacing the organic radicals with molecular metal complexes - which would require new chemical methods. It therefore seems that chemists hold the key to technologies and scientific discoveries involving magnetic graphene.

FERNANDO LUIS \& EUGENIO CORONADO 